\title{
Energy Cost Forecasting for Event Venues
}

\author{
Andrea Zagar, Katarina Grolinger, Miriam Capretz \\ Department of Electrical and Computer Engineering \\ Western University \\ London, ON, Canada N6A 5B9 \\ \{azagar2, kgroling, mcapretz\}@uwo.ca
}

\author{
Luke Seewald \\ London Hydro \\ London, ON, Canada N6A 4H6 \\ seewaldl@londonhydro.com
}

\begin{abstract}
Electricity price, consumption, and demand forecasting has been a topic of research interest for a long time. The proliferation of smart meters has created new opportunities in energy prediction. This paper investigates energy cost forecasting in the context of entertainment event-organizing venues, which poses significant difficulty due to fluctuations in energy demand and wholesale electricity prices. The objective is to predict the overall cost of energy consumed during an entertainment event. Predictions are carried out separately for each event category and feature selection is used to select the most effective combination of event attributes for each category. Three machine learning approaches are considered: k-nearest neighbor (KNN) regression, support vector regression (SVR) and neural networks (NN). These approaches are evaluated on a case study involving a large event venue in Southern Ontario. In terms of prediction accuracy, KNN regression achieved the lowest average error. Error rates varied greatly among different event categories.
\end{abstract}

Index Terms - energy cost forecasting, demand forecasting, machine learning, smart meters, prediction methods

\section{INTRODUCTION}

Predicting electrical energy consumption, demand, and price has been an active research and industry topic for more than a decade. Energy consumption forecasting on a national or regional level has played a major role in planning electrical production capacity. Recent advances in smart metering devices that collect, measure, and communicate energy consumption information have provided businesses and organizations with the opportunity to develop new ways of tracking and analyzing their energy usage, identifying savings potential, and forecasting their future energy usage.

An initiative known as Green Button has emerged as a facilitator for building energy-related applications and services [1]. This initiative provides utility customers standardized access to their past electrical energy data and the ability to consent to automatically have their utility share that data with third parties. Knowing past energy consumption information as provided by Green Button establishes the foundation for predicting future energy consumption, but is not sufficient for estimating the associated energy cost. This is emphasized in the case of commercial customers, who are often billed based on consumption (total amount of energy used), demand (rate of consumption), and market price. While activities may be consistent in their energy usage, their energy cost may vary greatly depending on the overall market energy demand and the market wholesale electricity prices. As well, there exist different pricing models for commercial customers that vary depending on geographical location and utility provider. Most, but not all of these pricing models calculate cost based on market wholesale prices and energy demand [2].

Predicting energy cost for commercial customers is altogether very difficult due to large fluctuations in the market electricity prices [3]. Market prices are directly correlated to how much energy is being consumed by the rest of the region [4]. The main driving factor for the electricity market price is the balance between demand and supply. Because the price is affected by demand, price prediction is closely related to market demand prediction.

This paper is concerned with large commercial customers, specifically event-organizing venues including sports arenas, concert halls, theatres, and conference centers. A relevant feature for such customers is to determine the electricity cost on the event level, that is, for a specific game or performance. The cost on the event level is important because it impacts the price that the venue owner charges event organizers for use of the facility.

The study has been developed in collaboration with Spectra Venue Management at Budweiser Gardens in London, Ontario. At present EventAssist, a software application, determines the cost of historical events by taking into account event electricity consumption and demand, monthly peak demand, global adjustment, and historical market prices. Although, the application already includes electricity consumption forecasting, it is not capable of estimating the overall energy cost of an event.

A possible approach for energy cost prediction is to forecast individual components including consumption, demand, price, and global adjustment, and then calculate the overall electricity cost. This approach is very challenging as it involves forecasting a number of components, electricity price probably being the hardest one to predict. However, this study takes a different approach; the energy cost of a future event is estimated based on the energy cost of past events, which are provided by the EventAssist application. This approach avoids the need to forecast individual cost components including demand and price. By relying only on past event attributes and cost to predict future event cost, the proposed approach avoids the need to forecast individual cost components (energy price, consumption, demand, global adjustment), which are difficult to predict. Moreover, the proposed approach aims to predict a single attribute (cost) as opposed to predicting several

\footnotetext{
A. Zagar, K. Grolinger, M.A.M Capretz, L. Seewald, Energy Cost Forecasting for Event Venues, Proc. of the IEEE Electrical Power \& Energy Conference, 2015. (C) 20115 IEEE. Personal use of this material is permitted. Permission from IEEE must be obtained for all other uses, in any current or future media, including reprinting/republishing this material for advertising or promotional purposes, creating new collective works, for resale or redistribution to servers or lists, or reuse of any copyrighted component of this work in other works
} 
components and calculating the overall cost. This in turn reduces model complexity.

Even though this work focuses on a specific type of consumer, specifically event venues, the approach can be adapted to other scenarios where the energy consumption is impacted by operating schedule and activity type, such as schools, offices, and hotels.

This study explores the use of three machine learning approaches to predict event cost: k-nearest neighbour (KNN) regression, support vector regression (SVR), and feed-forward neural networks (FFNN). The three approaches generate varying prediction error rates, whose magnitude is dependent on the event category. KNN regression generated the lowest average error rate of the three approaches.

The rest of this paper is organized as follows: Section II introduces KNN, SVR, FFNN, and performance metrics, while Section III reviews related work. The methodology is presented in Section IV and an evaluation in Section V. Finally, Section VI concludes the paper.

\section{BACKGROUND}

This section introduces the three machine learning approaches used in this study - k-nearest neighbour regression, support vector regression and neural networks and describes the performance metrics used to compare the prediction models.

\section{A. K-Nearest Neighbour Regression}

$\mathrm{K}$-nearest neighbour (KNN) regression [5] is a basic machine learning algorithm that can be used to predict new data values based on past data. A typical KNN search problem has a reference, or training, set of data points and a query point, which is the data point to be predicted. To determine the k-nearest reference points, distances are computed from every reference point to the query point. Distance functions such as Euclidean and Manhattan [5] are frequently used in this type of algorithm. Once the k-nearest neighbours of the query point are identified, as depicted in Fig. 1, their mean is calculated and assigned as the new value of the query point.

In the case of this study, the set of reference points correspond to events from the past two years and the query point represents a future event. The value being averaged and predicted is a single event energy cost.

\section{B. Support Vector Regression}

Support vector machines (SVM) are supervised learning systems that use a high dimensional feature space to yield prediction functions that are imposed on a subset of support vectors [6]. SVM is primarily used for classification. A

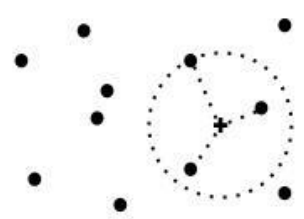

Figure 1. $\mathrm{A} \mathrm{k}=3 \mathrm{KNN}$ search problem; the query point is a plus sign, the reference points are circular dots, and the large circle expresses the distance to the third-farthest reference point from the query point specific form of SVM known as support vector regression (SVR) uses the same principles as SVM, but is modified to use regression rather than classification.

In SVR, support vectors are training samples that lie near the boundary of $\varepsilon$-tubes, which mark a certain threshold or margin of tolerance as observed in Fig. 2. The model produced by SVR only depends on a subset of the training data because observations that are close to the model prediction (within the threshold marked by the $\varepsilon$-tubes) are ignored.

\section{Neural Networks}

Neural networks (NN) are a group of machine learning models influenced by the inner workings of the human brain. They consist of interconnected neurons, or nodes, and have the ability to approximate nonlinear relationships between the input variables and output of a complicated system. Feed Forward Neural Networks (FFNN) are one of the most frequently used NNs for energy forecasting [8] and were chosen for this study.

As shown in Fig. 3, a feed forward neural network is composed of an input layer, one or more hidden layers of neurons, and an output layer. Each layer contains a chosen number of neurons, which are then individually interconnected with adaptable weighted connections to neurons in the succeeding layer (with the exception of the output layer). The output of each neuron in the hidden layer is determined using (1):

$$
f_{j}(x)=\varphi\left(\sum_{i=1}^{n} w_{i j} x_{i}+\theta_{i}\right)
$$

where $f_{j}(x)$ is the output of the $j$ th neuron, $\varphi$ is a transfer function (such as a Gaussian or sigmoid function), $x_{i}$ is the $i$ th input to the neuron, $w_{i j}$ is the connection weight between the $i$ th neuron in the input layer and the $j$ th neuron in the hidden layer, and $\theta_{i}$ is the bias or threshold. The neurons in the output layer also have weighted connections, exclusively with the last hidden layer in the network.

Training the network involves adjusting the weights between neurons so that the neural network can produce desirable results when given a set of inputs. A variety of training algorithms can then be used to minimize the network error function. This study uses a feed forward network with a single hidden layer and back-propagation learning algorithm.

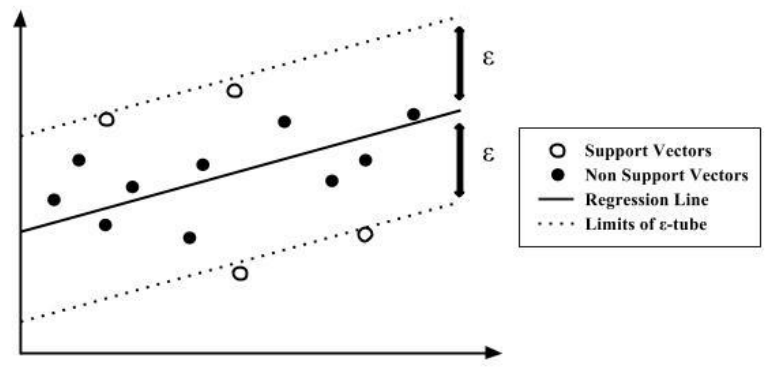

Figure 2. An example of SVR; this study uses non-linear SVR but linear is used for visualization purposes, adapted from [7] 


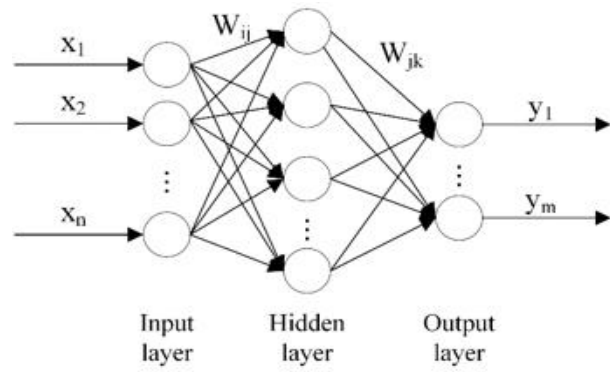

Figure 3. Feed forward neural network

\section{Performance Metrics}

Mean absolute percentage error (MAPE) [9] is the performance metric used in this study. It expresses the prediction accuracy of a forecasting method as a percentage, and is calculated as follows:

$$
M A P E=\frac{1}{n} \sum_{i=1}^{n} \frac{\left|y_{i}-\hat{y}_{i}\right|}{y_{i}} \times 100
$$

where $n$ is the number of observations, $y_{i}$ is the actual cost, and $\hat{y}_{i}$ is the predicted cost of the event.

\section{RELATED WORK}

A large number of research studies and industry projects have addressed various methods of electricity consumption, demand, and price prediction. A general collective agreement has been found between studies stating that price forecasting in energy markets is crucial for market participants in planning their operations, managing risk, and maximizing benefits [10]-[11].

Electricity market price is difficult to predict due to its high volatility caused by a variety of factors including volatility in fuel price, load uncertainty, and generation or transmission outages [11]. Moreover, price spikes routinely occur in the market, but are difficult to predict [12].

In the domain of market price prediction, the focus has been on short timeframes, especially day-ahead prediction [13], [10], [11]. Several popular machine learning techniques have been used in electricity price forecasting, but neural networks appear to be the most dominant. Many studies focused on developing prediction techniques based on variations of neural networks [13], [11], [14]. Examples of other machine learning approaches for price forecasting include Autoregressive Integrated Moving Average (ARIMA) with NN [15], and Recursive Dynamic Factor Analysis (RDFA) with Kalman filter [16]. Chen et al. [14] are concerned with lowering computation time for training prediction models; they apply extreme learning machines in their prediction model.

Market demand has a large influence on electricity pricing and therefore it has been accounted for in the price prediction models [17], [11]. Motamedi et al. [17] recognized the importance of studying the consumers' decisions and reactions when creating a forecasting framework. Consequently, they proposed a joint price and demand prediction. Singhal and Swarup [11] proposed an approach which relies on predicted demand values to forecast electricity price.
Similarly to price prediction, consumption and demand prediction has been carried out using different machine learning approaches including $\mathrm{NN}$ [18], SVR [7], and clustering models [19]. NN and SV-based models appear to be the dominant approaches in consumption prediction; they have been reviewed in the work of Ahmad et al. [20].

Our study differs from the reviewed works in two main aspects. Firstly, instead of forecasting energy consumption, demand, or price, our study focuses on predicting the overall electricity cost for events. Secondly, most of the aforementioned studies address short or medium-term (hours, days) forecasting, especially price prediction in concerned with very short timelines. In contrast, our study considers long-term prediction (several months) as the predicted cost should be known during venue booking negotiations.

\section{METHODOLOGY}

This section introduces the data set and describes the analyses and preparation that occurred before the machine learning approaches were applied. It also outlines feature selection and the model building.

\section{A. Data Set}

The data set includes energy consumption, cost of past events, and event attributes. Hourly event consumption data is obtained through Green Button. Preparations for events typically start hours before events and result in increased energy consumption. Thus, each event has a setup time, when the facility starts preparing for the event, and a teardown time, when the facility has completed clean-up after the event has finished. Therefore, hourly interval data is used to capture consumption for the entire span of the event including a setup and teardown time.

Cost of past events is obtained through the EventAssist application. This application breaks down the monthly electricity cost and attributes cost to individual events. The event cost is comprised of two primary components, namely consumption and demand. The consumption component is calculated using event consumption data, historical electricity prices and historical global adjustment. The demand component is determined by the distributed monthly demand cost to individual event proportional to the event demand peak.

The next set of variables included is event attributes together with the event schedule. Only variables that are known (or can be easily inferred) at the time of the event booking at the event facility are used for cost prediction. Variables such as weather forecast and number of event attendees are not included as they itself would require forecasting and can be of limited accuracy for longer timeframes.

Therefore, to account for temperature changes and seasons, this model uses day of the year as an input. This prevents weather forecasting errors from affecting energy prediction error. It is possible to include other variables as inputs; however, using them would introduce external error into the cost predictions. Any variables that are represented with categories or classes must be converted to a numerical 
scale in order for them to be used in the machine learning approaches. Specifically, the following event attributes and schedule variables are considered:

- Year: The year that the event takes place.

- Day of year: The day of the year, from 1-365, that the event takes place.

- Hour of event start: The hour of the day during which the event starts.

- Event duration: The time, in hours, from the beginning of the event itself to its end.

- Total duration: The time, in hours, from event setup to event teardown.

- Event category: The event category (e.g. hockey, basketball, theatre, etc.).

- Venue configuration: The event facility has a number of venue configurations that vary from event to event (e.g. full ice rink or full concert stage).

Not all input variables for each event category are used in the machine learning algorithms; this will be explained in detail in the Feature Selection section.

\section{B. Data Preparation and Analysis}

The predictions are performed separately for each event category, that is, separate prediction models are built for each category. Therefore, the data set is first separated into event categories. They include the following: Hockey, Basketball, Figure Skating, Ice Show, Dirt Track, Wrestling, Theatre, and Concert. In addition to the event attributes described previously, other attributes are manually added to specific event categories as necessary; for example, genre has been added to all Concert events and sub-category has been added to all Hockey events (e.g. regular season and playoffs).

Each event category is then analyzed individually using different graphs. Graphing event attributes against each other provides insight on trends and patterns that exist within the data. The pair of event attributes that were of most interest when beginning data analysis was energy consumption and cost. As shown in Fig. 4, a slight correlation exists between the two, as well as a notable difference. This is explained by the fact that cost varies not only depending on consumption, but also depending on the time of year and current market prices. Because of price volatility, variations in event energy cost are much larger than variations in energy consumption.

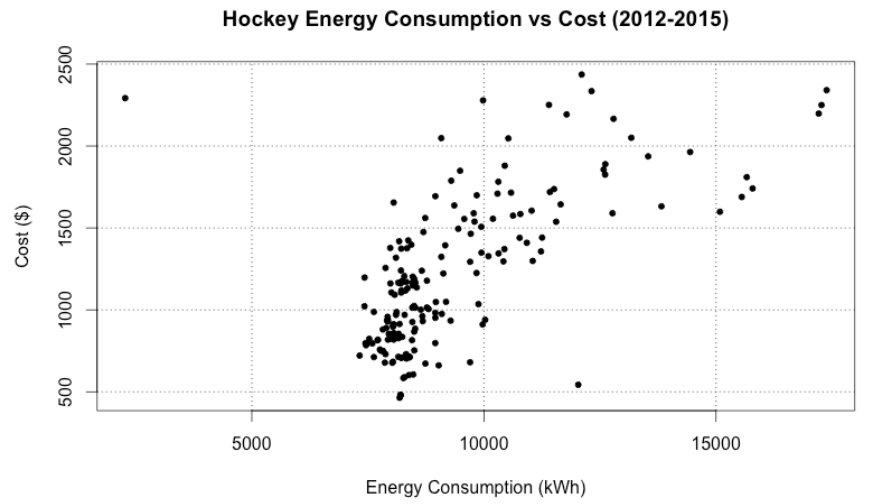

Figure 4. Energy consumption versus cost for the Hockey event category

\section{Feature Selection}

On account of reducing model complexity and training time, two feature selection approaches are taken to determine the optimal set of variables needed to train the cost prediction models. The two approaches are correlation matrices and recursive feature elimination (RFE). The training data used in the algorithms consists of all available input variables. Of the two approaches, RFE was primarily used and correlation matrices were briefly used for clarification in case RFE could not work properly due to data set anomalies.

The RFE algorithm focuses on generating a set of predictors that represent the optimal set of input variables. The specific version of the algorithm chosen for this study uses a random forest function to create the RFE model. 10-fold cross-validation is applied to obtain performance estimates that reflect the variation that occurs in feature selection.

Training data in RFE is used for several purposes, including predictor selection, model fitting and performance evaluation. If the training set is not large enough, especially in relation to the number of training data points, then the algorithm may not perform correctly. This occurred with two event categories, Dirt Track and Wrestling, since each had less than 10 data samples. Overall, the algorithm provided favourable results that were used in the cost prediction models in the next step.

The performances of different number of input variables for Basketball, as presented in Fig. 5, are plotted to provide a visualization of the algorithm results. It shows that based on the RFE calculations, using a subset of 2 variables (Day of Year and Year, which are not named in Fig. 5) is the most optimal solution.

\section{Model Building}

After the optimal sets of input variables are identified using feature selection, the data for each event category must be split into training and testing sets to prepare for model training. In the machine learning approaches, the training set is used for model training and selection, while the testing set is for evaluation only after the model has been created. The quality of the model cannot be evaluated using the same data used for fitting the model, or else it will be unclear whether or not the model is over-fitting [6]. In this study three machine learning algorithms are used to build the prediction model: KNN, SVR, and FFNN.

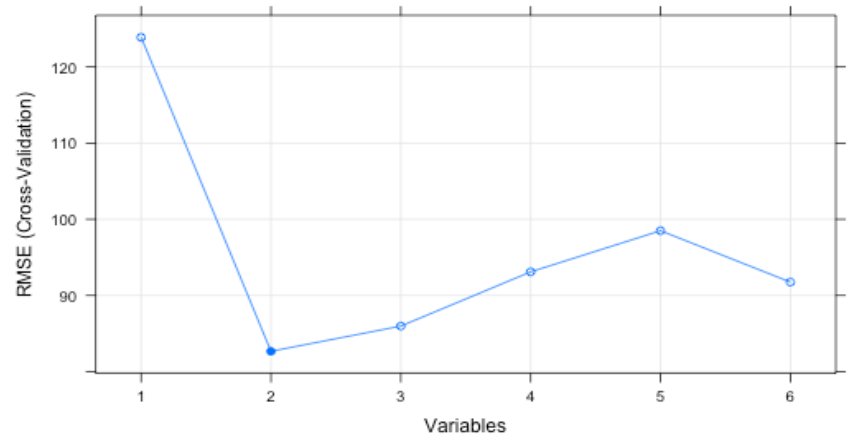

Figure 5. Performance of input variable subset sizes for the Basketball event category 


\section{Evaluation}

This section outlines the empirical data sets and implementation, describes the model building process, and discusses the results and findings of this study.

\section{A. Empirical Data Sets and Implementation}

The proposed approach has been evaluated on Budweiser Gardens, a large event venue located in London, Ontario. This venue hosts a variety of events, including professional sports such as hockey and basketball, and entertainment shows such as concerts and large theatre productions.

Event cost data was obtained through the EventAssist application, which uses Green Button data in its cost calculations. Event attributes and schedules are also obtained from the EventAssist database. Data spans from January $1^{\text {st }}$, 2012 to July $31^{\text {st }}, 2015$. Events were sorted into predetermined categories controlled by EventAssist.

In this study, training data for each event category encompasses all events occurring in 2013 and 2014. Testing data includes events occurring in 2015, from January $1^{\text {st }}, 2015$ to July $31^{\text {st }}, 2015$. For the event categories that had training data but no testing data, which include Figure Skating, Dirt Track and Wrestling, prediction models were created using each machine learning approach but no predictions were made. Consequently, the accuracy of the proposed approach for those event categories could not be evaluated.

The prediction models were implemented in the $\mathrm{R}$ language [21]. In specific, the KNN regression models were implemented using the "caret" package, the SVR models using the "e1071" package, and the NN models using the "RSNNS" package.

\section{B. Model Building}

The cost prediction models used in this study required a significant amount of preparation. Before fitting (i.e. creating) any of the models, it is important to ensure that the datasets for each event category are separated into training and testing sets, as outlined previously. All three machine learning algorithms in this study use the same training and testing sets for each event category to ensure uniformity between models of the same event category and to enable their comparison.

Depending on the algorithm, the data sets were normalized before applying the machine learning approaches, or the learning approach performed normalization internally. Normalization is important because it approximately equalizes the ranges of the input variables and allows them to each have the same effect when computing similarity [22].

After the necessary data is normalized, the model is fitted. 5-fold cross-validation is implemented for all algorithms during model fitting. Each event category has three separate models, each corresponding to a different machine learning algorithm: KNN, SVR, and FFNN.

Next, the testing data set is fed through the built model to generate event cost predictions. To evaluate model accuracy, the predicted cost is compared to the actual event costs using the mean absolute percentage error (MAPE) as described in (2). Using the resulting MAPE for each learning algorithm, the most optimal algorithm can be chosen for each event category.

K-nearest neighbour regression is the first machine learning approach explored in this study. Since the KNN model training function from the "caret" package does not automatically scale and centre data, the training and testing sets are normalized in the preparation step. KNN only has one parameter to vary, which is the number of nearest neighbours $k$. The values of $k$ chosen for testing ranges from $k=1$ to $k=$ 25 . During the model training process, the MAPE is taken for each subsequent value of $k$, and after the last value is tested, the model with the lowest MAPE is chosen as the final model. The value of $k$ in the final model varies for each event category. Overall, the model training process for $\mathrm{KNN}$ is very fast compared to the two other algorithms.

Support vector regression is the second approach explored in this study. The model training function for this approach ("e1071" package) internally normalizes the training and testing data, so no additional data manipulation was needed. SVR requires two parameters, gamma and cost. Gamma is a kernel function parameter and cost is a penalty parameter on the training error [23]. Gamma values from $10^{-8}$ to $10^{1}$ are tested, and for each gamma value, cost values from $10^{-3}$ to $10^{6}$ are tested. The step for both values is a multiplication by 10 . After all iterations are completed, the model with the lowest MAPE is chosen as the final model. Overall, the model training process for SVR is slower than KNN and comparable to $\mathrm{NN}$.

Neural networks are the last approach explored in this study. Like KNN regression, the model training function for this approach ("RSNNS" package) does not automatically scale and centre data, so the training and testing sets are normalized in the preparation step. With $\mathrm{NN}$ only one parameter is optimized - the number of hidden neurons. The range of values tested for this parameter is from 1 to 10. Five runs are completed for every iteration of cross-validation, because models may generate different error rates due to NN getting trapped in local minimum. The goal is to find the model that has the potential to produce the lowest error rate. Overall, the model training process for NN was significantly slower than KNN and comparable to SVR.

\section{Results and Findings}

Fig. 6 shows the MAPE values that each machine learning algorithm achieved on the testing set for each event category. As shown in the graph, the error rate for each machine learning approach is dependent on the event category. Basketball has similar error rates for all three models, while Ice Show has a higher error rate that varies depending on the machine learning algorithm. As Basketball has a more predictable yearly schedule, it is easier to predict future costs compared to Ice Show, where there is little evidence of a yearly pattern for when events occur.

Fig. 6 also shows the relative performances of each machine learning algorithm. KNN regression and NN perform somewhat similarly, while SVR is slightly more variant than the other two. 


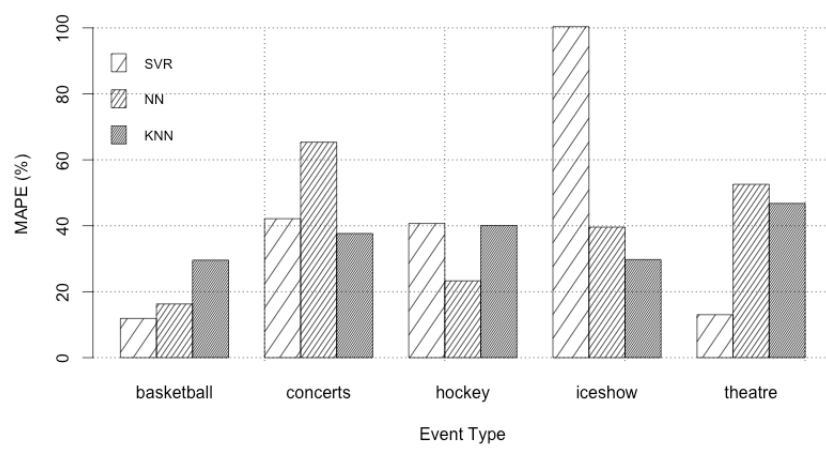

Figure 6. MAPE values for each machine learning approach for each event category

Some of the prediction error rates as shown in Fig. 6 are quite high; for example, in case of concerts all algorithm error rates are over $35 \%$. This can be explained by a large variance among different concerts; a small intimate concert will use much less electricity than a large rock music production. Although the error rates are relatively high, the proposed approach establishes the first step towards predicting electricity cost on an event level. Moreover, this study reveals the need to establish additional attributes to better describe events in terms of their energy consumption.

The actual cost and the predicted cost generated by each machine learning approach are graphed for each event category. An example for Hockey event category is shown in Fig. 7. It shows the slight differences between the predictions with different algorithms; $\mathrm{NN}$ tends to predict higher than KNN, for example. The right-hand side of the graph does not include actual cost data to compare the predictions to because the second half of the year has not occurred yet. When used for cost predictions in the future, the prediction models will produce data points without actual costs for validation.

\section{CONCLUSION}

Electricity consumption, demand, and price prediction has been a topic of research interest for a long time. Advances in smart metering devices have provided opportunity to develop new predictions approaches. However, the prediction remains a big challenge; market price is especially difficult to predict due to its volatility.

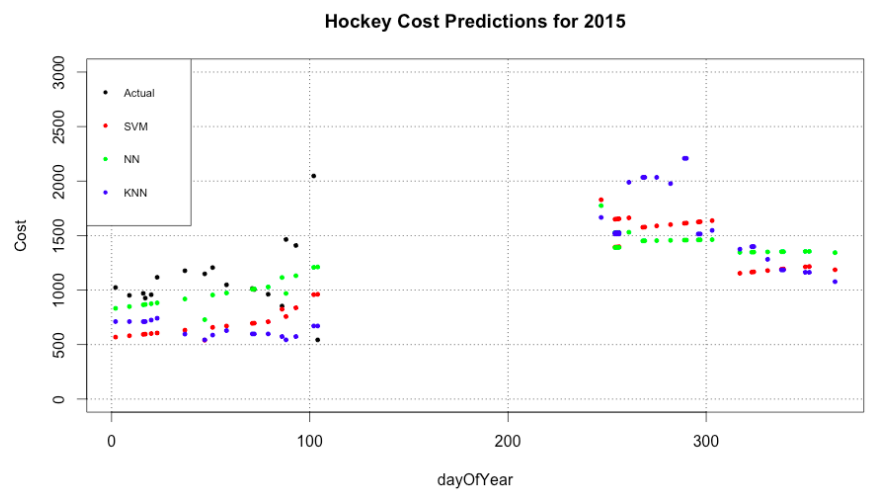

Figure 7. Cost predictions for the Hockey event category using all three prediction models
This study is concerned with commercial customers; specifically event venues, such as arenas or concert halls. The goal is to predict the overall cost of energy consumed by an event. This study explored the potential of using past event cost data and event attributes to estimate future event costs and highlighted the feasibility of doing so using the machine learning approach. Three machine learning algorithms were considered: KNN regression, SVR and NN. Of the three, $\mathrm{KNN}$ achieved the lowest average error rate for prediction accuracy. It was observed that the MAPE value for a machine learning approach depends strongly on the event category, as some categories are more difficult to predict than others.

Future work will investigate a more diverse range of input variables such as day of the week and electrical equipment in order to improve prediction accuracy. The approach will be evaluated on other event venues such as conference facilities and larger arenas. In addition, the use of 15-minute interval data rather than hourly data will be considered.

\section{ACKNOWLEDGMENT}

This research has been partially supported by the Ontario Centres of Excellence project, "Big Data Analytics for Energy Conservation in the Green Button Initiative". The authors would like to thank London Hydro for supplying industry knowledge, the Green Button platform and the data used in this study. They also would like to thank Budweiser Gardens for providing valuable data for this project.

\section{REFERENCES}

[1] US Department of Energy, "Green Button." http://energy.gov/data/green-button.

[2] Y. Wang and L. Li, "Time-of-use electricity pricing for industrial customers: A survey of US utilities," Applied Energy, vol. 149, pp. 89103, 2015

[3] C. Wan, Z. Xu, Y. Wang, Z. Y. Dong, and K. P. Wong, "A Hybrid Approach for Probabilistic Forecasting of Electricity Price," IEEE Transactions on Smart Grid, vol. 5, no. 1, pp. 463-470, 2014.

[4] Independent Electricity System Operator, "Electricity Pricing in Ontario, Large Electricity Consumers." http://www.ieso.ca/Pages/Ontario.

[5] D. H. Pandya, S. H. Upadhyay, and S. P. Harsha, "Fault diagnosis of rolling element bearing with intrinsic mode function of acoustic emission data using APF-KNN," Expert Systems with Applications, vol. 40, no. 10, pp. 4137-4145, Aug. 2013.

[6] T. Hastie, R. Tibshirani, and J. H. Friedman, The Elements of Statistical Learning: Data Mining, Inference, and Prediction, New York: Springer, 2009.

[7] R. K. Jain, K. M. Smith, P. J. Culligan, and J. E. Taylor, "Forecasting Energy Consumption of Multi-Family Residential Buildings using Support Vector Regression: Investigating the Impact of Temporal and Spatial Monitoring Granularity on Performance Accuracy," Applied Energy, vol. 123, pp. 168-178, 2014.

[8] R. Ž. Jovanović, A. A. Sretenović, and B. D. Živković, "Ensemble of Various Neural Networks for Prediction of Heating Energy Consumption," Energy and Buildings, vol. 94, pp. 189-199, 2015.

[9] R. J. Hyndman and A. B. Koehler, "Another look at measures of forecast accuracy," International Journal of Forecasting, vol. 22, pp. 679-688, 2006.

[10] P. Mandal, T. Senjyu, N. Urasaki, T. Funabashi, and A. K. Srivastava, "A Novel Approach to Forecast Electricity Price for PJM Using Neural Network and Similar Days Method," IEEE Transactions on Power Systems, vol. 22, no. 4, pp. 2058-2065, Nov. 2007.

[11] D. Singhal and K. S. Swarup, "Electricity price forecasting using artificial neural networks," International Journal of Electrical Power \& Energy Systems, vol. 33, no. 3, pp. 550-555, Mar. 2011. 
[12] J. H. Zhao, Z. Y. Dong, X. Li, and K. P. Wong, "A Framework for Electricity Price Spike Analysis with Advanced Data Mining Methods," IEEE Transactions on Power Systems, vol. 11, no. 1, pp. 376-385, 2007.

[13] M. Shafie-khah, M. P. Moghaddam, and M. K. Sheikh-El-Eslami, "Price forecasting of day-ahead electricity markets using a hybrid forecast method," Energy Conversion and Management, vol. 52, no. 5, pp. 2165-2169, May 2011.

[14] X. Chen, Z. Y. Dong, K. Meng, Y. Xu, K. P. Wong, and H. W. Ngan, "Electricity Price Forecasting With Extreme Learning Machine and Bootstrapping," IEEE Transactions on Power Systems, vol. 27, no. 4, pp. 2055-2062, Apr. 2012.

[15] J. C. R. Filho, C. M. Affonso, and R. C. L. Oliveira, "Energy price forecasting in the North Brazilian market using NN - ARIMA model and explanatory variables," in IEEE Symposium on Computational Intelligence for Engineering Solutions (CIES), 2014, pp. 171-175.

[16] H. C. Wu, S. C. Chan, K. M. Tsui, and Y. Hou, "New Recursive Dynamic Factor Analysis for Point and Interval Forecast of Electricity Prico Title," IEEE Transactions on Power Systems, vol. 28, no. 3, pp. 2352-2365, 2013.

[17] A. Motamedi, H. Zareipour, and W. D. Rosehart, "Electricity Price and Demand Forecasting in Smart Grids," IEEE Transactions on Smart Grid, vol. 3, no. 2, pp. 664-674, Jun. 2012.
[18] F. Ardakani and M. Ardehali, "Long-Term Electrical Energy Consumption Forecasting for Developing and Developed Economies Based on Different Optimized Models and Historical Data Types," Energy, vol. 65, pp. 452-461, 2014.

[19] W. El-Baz and P. Tzscheutschler, "Short-Term Smart Learning Electrical Load Prediction Algorithm for Home Energy Management Systems," Applied Energy, vol. 147, pp. 10-19, 2015.

[20] A. S. Ahmad, M. Y. Hassan, M. P. Abdullah, H. A. Rahman, F. Hussin, H. Abdullah, and R. Saidur, "A Review on Applications of ANN and SVM for Building Electrical Energy Consumption Forecasting," Renewable and Sustainable Energy Reviews, vol. 33, pp. 102-109, 2014.

[21] R Core Team, "R: A Language and Environment for Statistical Computing, Vienna, Austria: R Foundation for Statistical Computing," vol. 2014.

[22] S. Aksoy and R. M. Haralick, "Feature Normalization and likelihoodbased similarity measures for image retrieval," Pattern Recognition Letters, vol. 22, pp. 563-582, 2001.

[23] C. Huang and J. Dun, "A distributed PSO-SVM hybrid system with feature selection and parameter optimization," Applied Soft Computing, vol. 8, pp. 1381-1391, 2008. 